\title{
An approach to converge communication and RADAR technologies for intelligent transportation system
}

\author{
D. Kandar, S.N. Sur, D. Bhaskar, A. Guchhait, R. Bera and C. K. Sarkar ${ }^{1}$ \\ Sikkim Manipal Institute of Technology, Sikkim Manipal University, Majitar, Sikkim-737136, India \\ ${ }^{1}$ Dept. of Electronics and Telecommunication Engineering Jadavpur University, Kolkata-700032, India \\ kdebdatta@rediffmail.com
}

\begin{abstract}
The Intelligent Transport System (ITS) is an important and critical embedded system which should be carefully designed to fulfill the goals for safety and non-safety applications. ITS has been the umbrella under which significant efforts have been conducted in research, development, testing, deployment and integration of advanced technologies to improve the measures of effectiveness of national highway network. Efforts are being imparted towards a combined system of both communication and remote sensing technology. In the paper, the combined system construction principle of RADAR-location and information transmitting is considered. We modeled a communication and RADAR combined system in MATLAB considering WCDMA simulation model as reference. We considered moving target for simulation and it is detected at the RADAR receiver while the received information signal is processed through RAKE receiver to achieve low BER value. Thus the combined system is running successfully and satisfying both the requirements. This design can be implemented on reserved channel of Dedicated Short Range Communication (DSRC) for Intelligent Transport System (ITS) application.
\end{abstract}

Keywords: Intelligent Transport Systems (ITS); RADAR; WCDMA.

\section{Introduction}

In United States, there were 6279000 motor vehicle accidents that accounted for 41611 deaths in 1991. More dollars than any other cause of illness or injury are consumed for providing the health care regarding the treatment of crash victims. US department of Transportation has declared that the reduction of vehicular fatalities is its top priority. This is true for all other countries as well. Also demand for voice, data and multimedia services while moving in car increase the importance of Broadband wireless systems (Huang, 2006).

The Intelligent Transport System (ITS) adds information and communications technology to transport infrastructure and vehicles to improve safety and reduce vehicle wear, transportation times and fuel consumption etc. To provide potential benefits of ITS applications in a national highway network, a broad range of research and development efforts are being carried over under the umbrella of ITS Technology.

The first ITS research was developed by Japan in CACS (the comprehensive automobile traffic control system) in the year 1960s and 1970. Then it was stretched in US \& Germany respectively. In Japan, work on the road/automobile communication system (RACS) project, which formed the basis for our current car navigation system began in 1984. ITS is expected to become very big business in near future. Industry, like skysoft has become the major European player on command and control systems for the road sector, both for highways and cities. A wide variety of Japanese and international concerns are already involved in planning and development of ITS (Kahaner, 1996).

Research article

CIndian Society for Education and Environment (iSee)

"Intelligent transport system" http://www.indjst.org
Major technologies in ITS

ITS involves a broad range of wireless and wire-line communications-based information, control and electronics technologies. Short-range communications (less than 500 yards) can be accomplished using IEEE 802.11 protocols, specifically WAVE or the dedicated short range communications (DSRC) standard being promoted by the intelligent transportation society of America and the United States department of transportation. The US FCC has allocated $75 \mathrm{MHz}$ of spectrum in the $5.9 \mathrm{GHz}$ band $(5.8 \mathrm{GHz}$ for Europe and Japan) for DSRC to enhance the safety and productivity of the nation's transportation system.

Technological advances in telecommunications and information technology coupled with state-of-the-art microchip, RFID and inexpensive intelligent beacon sensing technologies have enhanced the technical capabilities that will facilitate safety benefits for motorist globally.

RADAR technology has been investigated for use on automobiles since the 1970s and has been employed for various functions on automobiles since the early 1980s (Belohoubek, 1987; Brus, 1987). Initial usage of microwave RADAR was for collision warning applications on commercial vehicles such as ambulances, buses and trucks. Prometheus, the ambitious European project that started in 1986, aimed to improve vehicle safety, efficiency and economy and was one of the main driving factors in the development of various types of sensors for automobiles, including millimeter wave RADAR. The advantage that RADAR sensors have over other types of sensors such as optical or infrared sensors, is that they perform equally well during the day, the night and in most 
weather conditions. RADAR can also be used for target identification and for detecting road conditions by making use of scattering signature information. The problems of clutter, multipath and interference are more severe (Kandar \& Bera, 2005). Proper technology in RADAR operation is to be implemented to mitigate those problems.
Fig. 1. DSRC channel scheme assigned in the United States

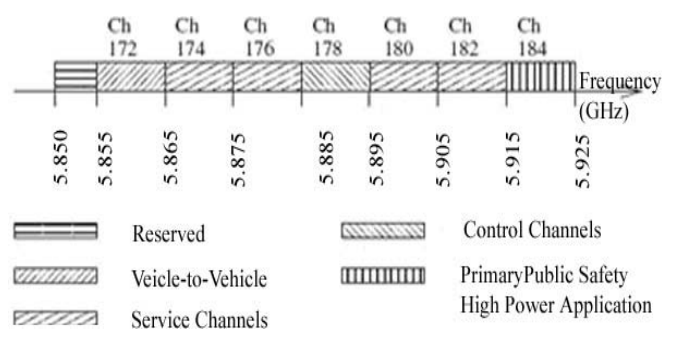

802.11 protocols, specifically WAVE or the DSRC standard being promoted by the intelligent transportation society of America and the United States department of transportation. DSRC, a sub-set of the RFID-technology offers communication between the vehicle and roadside equipment. This technology for ITS applications is working in the 5.9 $\mathrm{GHz}$ band (U.S.) or $5.8 \mathrm{GHz}$ band (Japan, Europe). Frequency allocation for DSRC channels are shown in Table 1. Dedicated short range

communication (DSRC) (Armstrong, 2008), standard being promoted by the intelligent transportation society of America and the United States department of transportation for

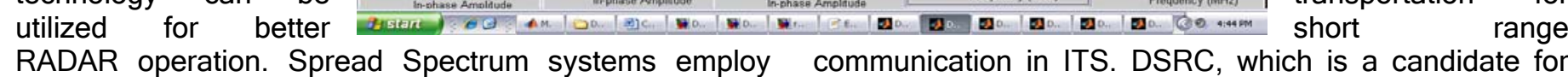
waveform similar to those of pulse compression RADAR.

\section{Advantages over conventional RADAR}

The design \& development of RCS Instrumentation RADAR at the low frequency band should be aimed for the additional benefits such as: local suppression of Interference due to coded RADAR waveform \& correlation of the received code, high level of rejection of multipath and higher reliability / efficiency \& low power consumption due to coding of the baseband pulses.

Accordingly, the waveform of choice will be Phase Coded Pulse Compression using digital techniques instead of linear frequency modulation (LFM) based analog techniques. Sometimes pulse compression RADARs have been called spread spectrum RADARs (Knott, 2004; Skolnik, 2007). Therefore, DSSS RADAR will be aimed for the development.

\section{Dedicated \\ communication}

short-range

Short-range communication can be accomplished using IEEE

Research article

CIndian Society for Education and Environment (iSee)
Fig. 3. Base band spreaded data generation at the transmitter side

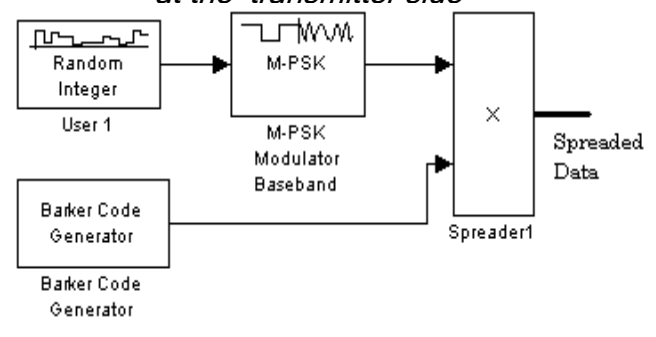

"Intelligent transport system" http://www.indjst.org use in a VANET offers the potential to effectively support in ITS. A number of wireless solutions were evaluated for use as the primary communication medium for DSRC (VSCC, 2005) which enables a new class of communication applications that will increase the overall safety and efficiency of the transportation system.

\section{DSRC as communication system for ITS}

First generation DSRC was used for toll collection system operates at $915 \mathrm{MHz}$ and has a transmission rate of $0.5 \mathrm{Mb} / \mathrm{s}$. The federal communication commission (FCC, 2002) allocated the $75 \mathrm{MHz}$ of bandwidth in the 5.9 $\mathrm{GHz}$ band for the second generation of DSRC. Since the allocation of the bandwidth, standardization bodies have been working on the implementation details of $5.9 \mathrm{GHz}$ DSRC. To reduce the traffic hazards, the North American DSRC standards program aims at creating an interoperable standard for use in the US, Canada and Mexico. Furthermore, $5.9 \mathrm{GHz}$ DSRC must have a low cost and be very 
scalable. In addition, the $5.9 \mathrm{GHz}$ DSRC should require no usage fee from the users to access the network.

Now same DSRC channel can be utilized for RADAR operation. The $5.9 \mathrm{GHz}$ DSRC spectrum is composed of 6 service channels and 1 control channel each having bandwidth of $10 \mathrm{MHz}$ as shown in Fig.1. The first (remaining) channel of DSRC (having $5 \mathrm{MHz}$ bandwidth) is reserved. This reserved channel $(5.850-5.855 \mathrm{GHz})$ can be used for remote sensing which involves spread spectrum based digital RADAR technology.

\section{RADAR as remote sensing device}

There are various kinds of sensors available for ITS applications as remote sensing devices. The remote sensing devices based on using sensors are pneumatic road tubes, inductive loops, magnetic sensors, piezoelectric sensors, video cameras, infrared lasers sensors, microwave (MW) RADARs and ultrasonic sensors (Mimbela \& Klein, 2000; Hsieh et al., 2006). Sensor will provide a specific mechanism of detecting vehicles and has its own advantages and disadvantages. Since user needs and classification conditions can differ, no sensors and corresponding techniques have proven to be the best for all possible applications (Mimbela \& Klein, 2000) (FHWA-PL-01-021, 2001).

RADAR technology has been investigated for use on automobiles since the 1970s and has been employed for various functions on automobiles since the early 1980 s (Belohoubek, 1987; Brus, 1987; Wegner, 1998; Gandhi \& Trivedi, 2006). RADAR is having the advantage of high detection range, high range resolution and lower algorithmic complexity with moderate hardware cost. The advantage that RADAR sensors perform equally well during the day, the night and in most weather conditions (Dixit \& Rafaelli, 1997; Gandhi \& Trivedi, 2006). Considering the physical size of the RADAR sensors most current millimeter-wave RADAR systems operate in the 76-77 $\mathrm{GHz}$ frequency range (Dixit \& Rafaelli, 1997; Wegner, 1998).

Though in various traffic management applications, roadside mounted and forward-looking frequencymodulated continuous wave (FMCW) and noise-

$\begin{aligned} & \text { Table 1. DSRC channel } \\
& \text { no. with operating } \\
& \text { frequency }\end{aligned}$
\begin{tabular}{|l|c|}
\hline $\begin{array}{l}\text { Channel } \\
\text { no. }\end{array}$ & $\begin{array}{c}\text { Frequency } \\
\text { (GHz) }\end{array}$ \\
\hline 172 & $5.855-5.865$ \\
\hline 174 & $5.865-5.875$ \\
\hline 175 & $5.865-5.885$ \\
\hline 176 & $5.875-5.885$ \\
\hline 178 & $5.885-5.895$ \\
\hline 180 & $5.895-5.905$ \\
\hline 181 & $5.895-5.915$ \\
\hline 182 & $5.905-5.915$ \\
\hline 184 & $5.915-5.925$ \\
\hline
\end{tabular}

Vol. 3 No. 4 (Apr. 2010)

ISSN: 0974- 6846
Fig. 4. Target model methodology

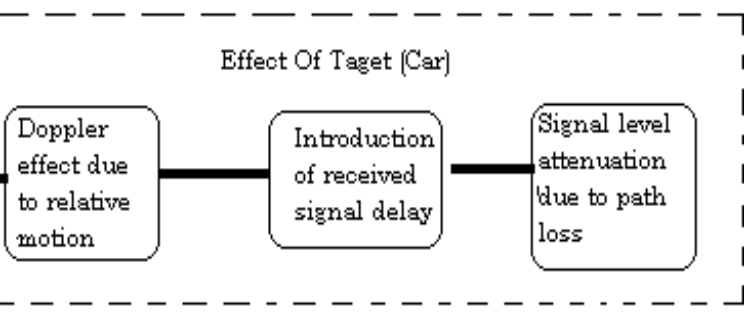

correlation RADAR units combined with continuous-wave $(\mathrm{CW}) \rightarrow$ doppler sensors are commonly used (Roe \&

Fig. 5. Target model for RADAR system

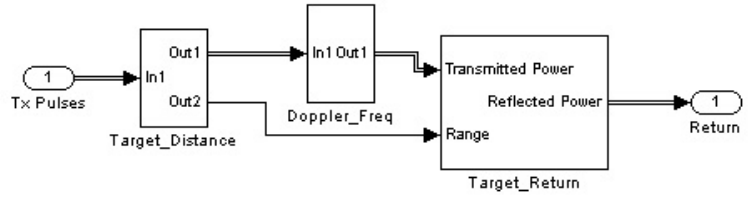

Target_Return

Hobson

1992;

Walton et al., 1997).

RADAR operation in a moving vehicle is very difficult because of ground clutter problem. Instead of using analog operation, digital RADAR operation will help in ITS.

\section{Combined RADAR System}

In the combined system of RADAR tracking detection of the targets and information transmitting on the two separate reserved DSRC channel. We modeled a communication and RADAR combined system in MATLAB considering WCDMA simulation model as reference.

The procedure to construct a combined channel for a RADAR target detection and digital communication system consists in the following.

- Construction of the RADAR and communication combined system. In this paper we use WCDMA physical layer simulation as reference. As in DSRC, we use 2 separate channels, 1 for RADAR purpose and other for communication.

- For both the communication and RADAR purpose we have used spread spectrum technique. For both the cases we have use 2 stage modulation techniques.

(a) QPSK modulation.

(b) Spread spectrum modulation using high data rate chip signal.

- Complex signal processing for noise immunity of the combined system.

- The estimation techniques of the signal power parameters for joint system of a RADAR- location and communication. In this paper to equalize the received 
Fig. 7. Simulation Model of Combined Receiver

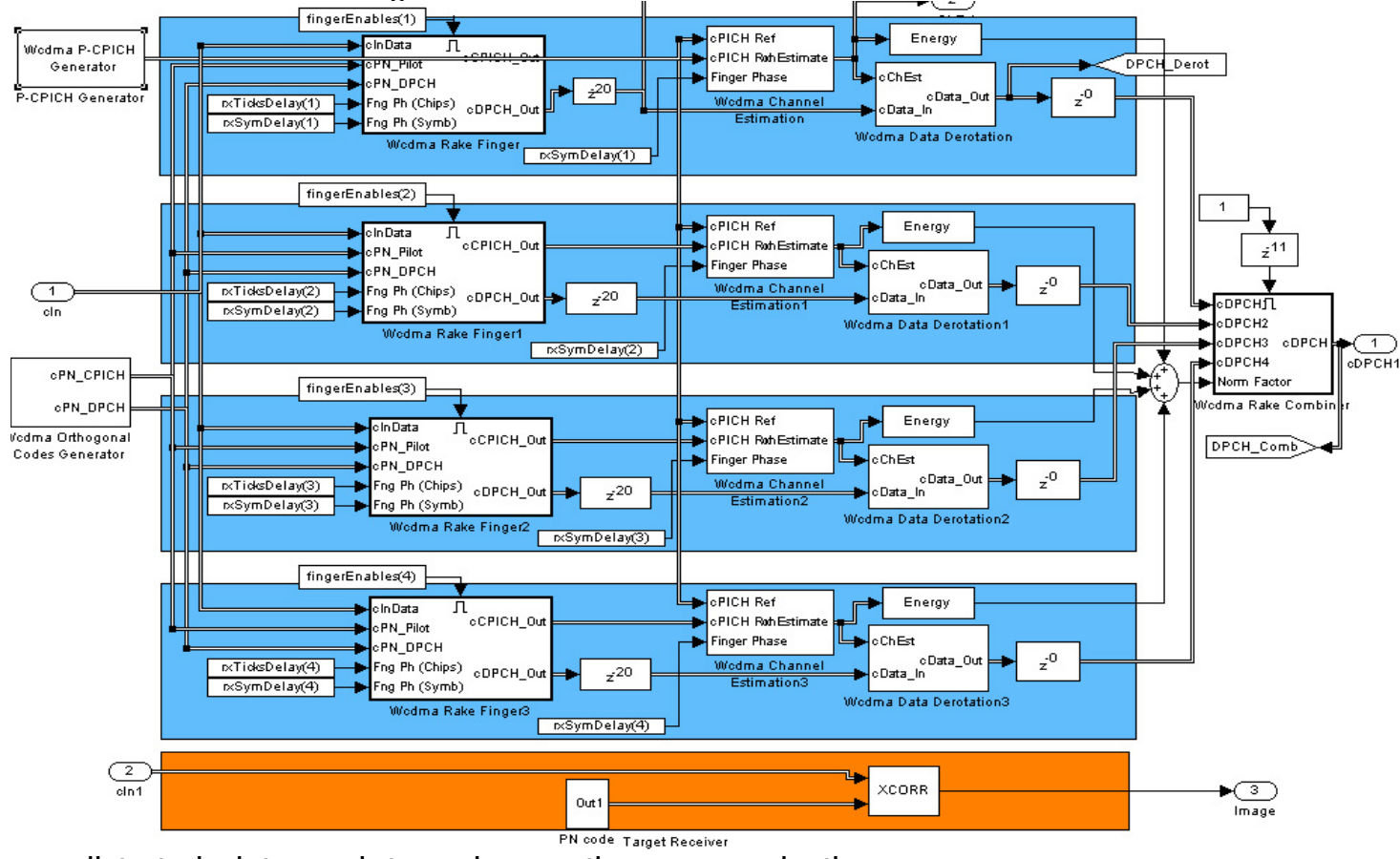

signal that does not significantly interfere with other signals. In our designed we used 13 bit Barker code as PN code and after spreading we have up converted it and transmitted it to the channel

Channel and target model:

In channel modeling we considered multipath channel. We introduce the relative motion between the cars by incorporating Doppler Effect to the transmitted signal by taking into account of motion of the car. We also successfully developed a target model. The transmitted signal gets reflected form the target and we measure the time in order to find its relative position. The schematic diagram of our designed target model is shown in Fig. 4. The block diagram of the target model and the simulation model is shown in Fig. 4 and Fig.5.

\section{Receiver}

In receive section of SS RADAR operation, correlation plays an important role. Same barker code has been used as PN code for correlation. The basic block diagram of SS RADAR receiver is shown in Fig. 6.

When the size of the target is fixed the normalized cross-correlation method can be directly applied for Target Tracking (Behrad et al., 2001). We use XCORR Simulink block in order to cross correlate the received signal with the transmitted signal. And find some interesting result showing the car movement.

Fig. 7 shows the combined RAKE receiver simulation model which includes RAKE processor (four blue blocks) for communication device as well as for RADAR device (orange block).

\section{Conclusion}

The demand of ITS encourage us to work for converging of communication and RADAR technology. DSRC is well established in communication system. We successfully simulated RADAR model combined with communication model. The MATLAB simulation model is able to calculate BER (Bit error rate), PER (Packet error rate) as well detect the target. The system is able to measure the target distance. It also can separate different targets placed $30 \mathrm{~m}$ apart.

\section{Transmitter}

In transmitter section of SS RADAR operation as shown in Fig. 3. Modulated waveform is modulated (spread) again over an expanded bandwidth wideband

Research article

CIndian Society for Education and Environment (iSee)
"Intelligent transport system" http://www.indjst.org
Kandar et al. Indian J.Sci.Technol. 
But we considered all aspects of RADAR operation in the base band level. As SS technology is implemented in DSRC, no extra channel is required for RADAR operation. Also unwanted scatterers are eliminated to a great extent. As the RADAR can be implemented in car its physical size is very important parameter for its commercial use. So the radio part of the RADAR should operate in higher frequencies like $90 \mathrm{GHz}$ or more. Other techniques like MTI can detect moving targets from ground clutter, even in the bad weather by virtue of the doppler radar return of the moving targets (Repesh, 2002; Dunn et al., 2004). RADAR can extract the doppler frequency shift of the echo produced by a moving target by noting how much the frequency of the received signal differs from the frequency of the signal that was transmitted.

\section{References}

1. Armstrong L (2008) Dedicated short-range communications project.

2. Behrad A, Shahrokni A and Motamedi SA (2001) Robust vision-based moving target detection and tracking system. In: Proc. Image \& Vision Computing Conf. (IVCNZ2001), Univ. of Otago, Dunedin, New Zealand, Nov. 26-28.

3. Belohoubek EF (1987) RADAR control for automotive collision mitigation and headway spacing. IEEE Trans. Veh. Technol. 31. Pp 88-89.

4. Brus E (1987) Vehicular RADAR: The ultimate aid for defensive driving. Microwaves \& RF. Pp 53-58.

5. Cooper GR and CD McGillem (1986) Modern communication and spread-spectrum, New York. McGraw-Hill.

6. Dixit $R$ and Rafaelli $L$ (1997) RADAR requirements and architecture trades for automotive applications in Proc. IEEE MTT-S Dig. 3. Pp 1253-1256.

7. Dixon RC (1984) Spread-spectrum systems, New York. John Wiley \& Sons.

8. DSRC (2005) Vehicle Safety Communications Consortium consisting of, BMW, Daimler-Chrysler, Ford, GM, Nissian, Toyota and VW. Vehicle safety communications project task 3 final report.

9. Dunn J, Bingham P, Charles A and Fowler B (2004) Ground moving target indicator RADAR ground moving target indicator RADAR and the transformation of U.S. War fighting Northrop Grumman Corp. pp 1-30.

10. FCC (2002) Amendment of the commission's rules regarding dedicated short-range communication service in the $5.850-5.925 \mathrm{GHz}$ band, fcc 02-302. Tech. rep., FCC.

11. Gandhi S and Trivedi M (2006) Pedestrian collision avoidance systems: A survey of computer vision based recent studies. Proc. of the IEEE ITSC 2006, IEEE Intell. Transp. Syst. Conf. Toronto, Canada.
12. Glisic SG and PA Leppanen (1995) Code division multiple access communications, Norwell, MA. Kluwer Academic Publishers.

13. Hanzo L, Yong LL, Kuan EL and Yen K (2003) Single and Multi-Carrier DS-CDMA Multy-User Detection, Space-Time Spreading Synchronisation and Standards. John Wiley \& Sons, $1^{\text {st }}$ edition.

14. Hsieh JW, Yu SH, Chen YS and Hu WF (2006) Automatic traffic surveillance system for vehicle tracking and classification. IEEE Trans. Intell. Transp. Syst. pp175-177.

15. Huang $X$ (2006) Smart antennas for Intelligent transportation systems. $6^{\text {th }}$ Int. Conf. on ITS telecommunications proc. pp 426-429

16. Kahaner (1996) ATIP Report: ITS (Intelligent Transport System).

17. Kandar D and Bera R (2005) Development of a imaging RADAR instrumentation system using DSUWB wireless technology. Proc. of XXVIII ${ }^{\text {th }}$ general assembly of int. union of radio science (URSI).

18. Knott EF (2004) RADAR Cross Section. Scitech publishing, Raleigh, NC.

19. Mimbela K and Klein LA (2000) A summary of vehicle detection and surveillance technologies used in intelligent transportation systems. Las Cruses, NM: The vehicle detector clearing house. http://www.fhwa.dot.gov/ohim/tvtw/vdstits.pdf

20. Repesh C (2002) Broadband FIR moving-target indicator RADAR filters for Low-PRF applications. VMEbus systems.

21. Roe $H$ and Hobson GS (1992) Improved discrimination of microwave vehicle profiles in Proc. IEEE MTT-S Int. Microw. Symp.

22. Scholrz RA (1977) Spread-Spectrum Concept. IEEE Trans. Commun. Vol 25, no. 8, pp. 748-755.

23. Richard C. Dorf et al., (1993) The electrical engineering handbook, CRC press, Pp 1563 - 1574.

24. Skolnik MI (2007) Introduction to RADAR systems. TATA McGraw-Hill Publication.

25. Torrieri DJ (1992) Principles of secure communication Systems, Norwood, Artech House.

26. Viterbi AJ (1995) CDMA principles of spreadspectrum communications, MA. Addison-Wesley.

27. Walton EK, Theron IP, Gunawan S and Cai L (1997) Moving vehicle range profiles measured using a noise RADAR in Proc. IEEE Antennas and Propag. Soc. Int. Symp.

28. Wegner J (1998) Automotive mm-wave RADAR: status and trends in system design and technology in. Proc. Instt. Elect. Eng. Colloquium Automotive RADAR \& Navigation Techn.
Research article

CIndian Society for Education and Environment (iSee)
"Intelligent transport system" http://www.indjst.org
Kandar et al. Indian J.Sci.Technol. 